\title{
Neonatal chlamydial pneumonia induces altered respiratory structure and function lasting into adult life
}

\author{
Madhulika Jupelli ${ }^{1}$, Ashlesh K Murthy ${ }^{1}$, Bharat KR Chaganty ${ }^{1}$, M Neal Guentzel $^{1}$, Dale M Selby ${ }^{2}$, \\ Margarita M Vasquez ${ }^{3}$, Shamimunisa B Mustafa ${ }^{3}$, Barbara M Henson ${ }^{3}$, Steven R Seidner ${ }^{3}$, Guangming Zhong ${ }^{4}$ \\ and Bernard P Arulanandam ${ }^{1}$
}

Respiratory dysfunction in adults has been correlated with neonatal Chlamydia trachomatis pneumonia in several studies, but a causal association has not been clearly demonstrated. In this study, we examined radial alveolar counts (RACs) by microscopy, and airway and parenchymal lung function using a small animal ventilator in juvenile ( 5 weeks age) and adult ( 8 weeks age) BALB/c mice challenged as neonates with Chlamydia muridarum (C. mur) on day 1 or day 7 after birth, representing saccular (human pre-term neonates) and alveolar (human term neonates) stages of lung development, respectively. Pups challenged with $C$. mur on either day 1 or 7 after birth demonstrated significantly enhanced airway hyperreactivity and lung compliance, both as juveniles (5 weeks age) and adults ( 8 weeks age), compared with mockchallenged mice. Moreover, mice challenged neonatally with Chlamydia displayed significantly reduced RACs, suggesting emphysematous changes. Antimicrobial treatment during the neonatal infection induced early bacterial clearance and partially ameliorated the Chlamydia-induced lung dysfunction as adults. These results suggest that neonatal chlamydial pneumonia, especially in pre-term neonates, is a cause of respiratory dysfunction continuing into adulthood, and that antimicrobial administration may be partially effective in preventing the adverse respiratory sequelae in adulthood. The results of our studies also emphasize the importance of prenatal screening and treatment of pregnant women for C. trachomatis in order to prevent the infection of neonates.

Laboratory Investigation (2011) 91, 1530-1539; doi:10.1038/labinvest.2011.103; published online 18 July 2011

KEYWORDS: airway hyperreactivity; antimicrobial treatment; infection during saccular and alveolar lung development; lung parenchymal changes; neonatal pulmonary chlamydial infection; respiratory sequelae in juvenile and adult life

Perinatal transmission of Chlamydia trachomatis has been reported to cause neonatal pneumonia within $4-6$ weeks after birth. $^{1-3}$ The infection is characterized by interstitial and peribronchiolar inflammation ${ }^{4}$ in the first few weeks of life leading to significant morbidity manifested as low grade fever and staccato cough unless intervened by antibiotic treatment. $^{5}$ It is given that prenatal screening and treatment of pregnant women for C. trachomatis in developed countries has been successful in largely reducing the incidence and morbidity due to neonatal pulmonary chlamydial infection (CDC, 2010 STD Guidelines; cdc.gov/ std/treatment/2010/chlamydial-infections.html). However, if infection of pregnant women is not detected and treated, several anomalies in lung function of children, including reduced total lung capacity and forced expiratory volume, have been correlated with the past clinical history of neonatal C. trachomatis pneumonia. ${ }^{6}$

Likewise, human respiratory infections with Chlamydia pneumoniae later in life (infant, childhood and adult) have been associated with induction, severity and exacerbations of wheezing, and asthma. ${ }^{7-9}$ In a recent study of Chlamydia muridarum (C. mur) infection of mice as neonates, infants or adults, followed 6 weeks later with sensitization and then challenge with ovalbumin, the early life (neonatal and infant), but not the adult infection with Chlamydia, led to enhanced allergic airways disease with features of asthma. Some of the problems associated with a better understanding of the role of the developmental stage of life and past, acute

\footnotetext{
${ }^{1}$ Department of Biology, South Texas Center for Emerging Infectious Disease, University of Texas at San Antonio, San Antonio, TX, USA; ${ }^{2}$ Department of Pathology, Wilford Hall Medical Center, San Antonio, TX, USA; ${ }^{3}$ Department of Pediatrics, University of Texas Health Science Center, San Antonio, TX, USA and ${ }^{4}$ Department of Microbiology and Immunology, University of Texas Health Science Center, San Antonio, TX, USA

Correspondence: Professor BP Arulanandam, Department of Biology, South Texas Center for Emerging Infectious Diseases, University of Texas at San Antonio, One UTSA Circle, San Antonio, TX 78249, USA.
}

E-mail: Bernard.arulanandam@utsa.edu

Received 20 October 2010; revised 23 May 2011; accepted 23 May 2011 
or chronic infection with C. pneumoniae or C. trachomatis in human respiratory dysfunction relates to lack of wellstandardized, validated, commercial diagnostic tests, and how to best interpret them ${ }^{10,11}$ as well as the need for greater data and detail on respective clinical manifestations of the infections. ${ }^{9,11}$ For example, the incidence of respiratory infection due to C. pneumoniae in adults was $0-17 \%$ and in pediatric patients $3-44 \%$ in one review ${ }^{10}$ of studies differing by location and diagnostic method. The rates of C. trachomatis conjunctivitis varied from 8 to $44 \%$ (average 15\%) and C. trachomatis pneumonia from 0 to $17 \%$ (average $7 \%$ ) in studies of infants exposed at birth in another review. ${ }^{12}$ Although the incidence of $C$. trachomatis pneumonia will be low in locales with vigorous prenatal screening and treatment programs, in developing countries without prenatal screening, the incidence of C. trachomatis pneumonia in exposed neonates may be high, as in studies in Kenya ${ }^{2}$ and in India. ${ }^{1}$ In a recent United States study by Webley et al, ${ }^{13}$ Chlamydiaspecific DNA was detected in $124(68 \%)$ of 182 pediatric patients with chronic respiratory diseases with $42 \%$ positive for C. trachomatis, $43 \%$ positive for C. pneumoniae and $18 \%$ positive for both. Although the greatest numbers of infected patients were in the age range of $0-2$, a significant number, including ones with cultivable organisms in BALF, were found in older age pediatric patients. The deficient understanding of the role of Chlamydia in respiratory dysfunction underscores a need for detailed evaluation of a cause-effect relationship and mechanisms involved in the induction of such chronic sequelae.

To this end, we recently established a mouse model to study the acute and chronic effects of neonatal infection with Chlamydia muridarum and demonstrated that 1-day-old pups challenged intranasally (i.n.) with the bacterium display interstitial- and bronchopneumonia, until 2 weeks after chlamydial challenge. ${ }^{14}$ In addition, we demonstrated that the IL-12/IFN- $\gamma$ axis has an important role in the inflammatory cellular recruitment into the lungs which, in turn, appeared to augment containment and clearance of the neonatal pulmonary chlamydial infection. ${ }^{15}$ Others have used a similar model and recently reported that the pulmonary chlamydial infection in neonatal mice led to chronic sequelae in adult life. This was apparent as an altered T-helper (Th) 1 type of immune response to an otherwise predominantly Th2 type response to the prototypic allergen ovalbumin, ${ }^{16}$ associated with exacerbated airway hyperreactivity (AHR) and allergic airway disease (AAD) ${ }^{16}$ It also was noted that the neonatal mouse infection per se might induce alveolar and bronchiolar dysfunction in adulthood. ${ }^{17}$

In this study, we characterized in detail the pathological sequleae occurring weeks after chlamydial clearance from the lungs, in respiratory structure and function induced by neonatal C. mur infection in $\mathrm{BALB} / \mathrm{c}$ mice. C. mur challenge at 1 or 7 days following birth (D1 and D7) was used to model the infection occurring in two different stages of lung development: saccular and alveolar, respectively. The chronic sequelae were then evaluated at 5 and 8 weeks after birth to represent the effects in adolescent and adult life, respectively. C. mur-challenged mice demonstrated greater AHR, emphysematous changes and decreased radial alveolar counts (RACs). The sequelae following challenge in D1 pups were more pronounced than those seen following challenge in D7 pups. Importantly, we found that administration of an antimicrobial, although efficacious in reducing bacterial burden and acute inflammation, only partially ameliorated the chronic sequelae in adult life following infection on an immature lung compared with infection on a matured lung.

\section{MATERIALS AND METHODS \\ Bacteria}

C. mur was grown in HeLa cell monolayers and purified as described previously. ${ }^{14}$ Briefly, the chlamydial elementary bodies (EBs) were harvested by lysing the infected HeLa cells using a sonicator (Fisher, Pittsburgh, PA), followed by purification of the EBs on renografin gradients. Aliquots of bacteria were stored at $-80^{\circ} \mathrm{C}$ in sucrose-phosphateglutamine buffer.

\section{Mice}

Adult (6-8 weeks old) BALB/c mice were purchased from Jackson Laboratory (Bar Harbor, ME). Mice were housed and bred at the University of Texas at San Antonio Animal Care Facility and provided food and water ad libitum. One and seven day old pups were infected and evaluations of respiratory structure and function were conducted at the age of 5 and 8 weeks. Animal care and experimental procedures were performed in compliance with the Institutional Animal Care and Use Committee guidelines.

\section{Intranasal Chlamydial Challenge}

Groups of 1- and 7-day-old BALB/c mice were challenged i.n. with approximately 100 inclusion-forming units (IFUs) of C. mur in $5 \mu \mathrm{l}$ of sterile PBS. Mock-challenged mice received sterile PBS. The pups were monitored daily for body weight and weaned at 3 weeks of age. Tests of lung function were performed at 5 weeks (juvenile stage) or 8 weeks (adult stage) using the FlexiVent, a small animal ventilator (SCIREQ, Montreal, Province of Quebec, Canada).

\section{Western Blot Analysis}

Groups of 1-day-old BALB/c mice were challenged with C. mur and rested for 7 days or 5 weeks before surfactant expression was analyzed by Western blot. Briefly, lungs were collected ( 7 days or 5 weeks post challenge), homogenized and protein concentrations were determined by the Bradford assay using bovine serum albumin as a standard. Equal amounts of proteins $(30 \mu \mathrm{g})$ were resolved by SDS-PAGE on $10 \%$ gels and transferred onto nitrocellulose membranes. Nonspecific binding sites were blocked by incubating the membranes with $5 \%$ low-fat dried milk in Tris-buffered saline-Tween 20 (TBST). The membranes were then probed 
for surfactant proteins overnight at $4{ }^{\circ} \mathrm{C}$ in $1 \%$ milk using antibodies specific for SP-A, SP-B, SP-C and SP-D (Santa Cruz Biotechnology Inc., CA). Membranes were then washed with TBST and further incubated with secondary antibody; goat anti-rabbit conjugated with horseradish peroxidase. The blots were developed and visualized using ECL reagents (Pierce, IL). The images were then analyzed using a phosphor-image analyzer and the protein concentrations expressed were determined by normalizing to $\beta$-actin.

\section{Antimicrobial Therapy}

Groups of 1- and 7-day-old BALB/c mice were challenged with $C$. mur and rested for 2 days before antibiotic treatment. Erythromycin (Sigma Aldrich, MI) was chosen from one of the drugs of choice based on CDC, 2010 STD Treatment Guidelines (cdc.gov/std/treatment/2010/chlamydialinfections.html) and the available literature, ${ }^{5}$ and was administered at $50 \mathrm{mg} / \mathrm{Kg} /$ day body weight into the peritoneal cavity every $12 \mathrm{~h}$ for 3 consecutive days. Lungs were collected before and after completion of antibiotic administration, homogenized and plated onto HeLa cell monolayers to measure bacterial burden, as described previously, by staining for chlamydial inclusions at $28 \mathrm{~h}$ after inoculation of the monolayers. ${ }^{14,18}$ Groups of pups were rested for 8 weeks and lung function was evaluated using the FlexiVent.

\section{Radial Alveolar Counts}

To quantify the number of alveoli, RACs were obtained as described by Emery and Mithal ${ }^{19}$ and validated by Cooney and Thurlbeck. ${ }^{20}$ Briefly, the mice were killed at the indicated times, a small midline incision was made on the ventral surface of the neck and the trachea was exposed by blunt dissection. The trachea was cannulated with a 27-guage needle, and the lungs were fixed with $10 \%$ neutral formalin at a constant pressure of $25 \mathrm{~cm}$ of $\mathrm{H}_{2} 0$. Following overnight incubation in neutral formalin, the lungs were embedded and serial mid-sagittal sections were made as described. The sections were then stained with hematoxylin and eosin (H\&E), and RACs performed as described. ${ }^{19,20}$ Basically, a perpendicular line was drawn from the respiratory bronchiole to either the pleura or the nearest connective tissue septum. Using low power images a minimum of fifteen lines were drawn for each lung (three sections per mouse) and the number of alveoli that intersected was counted for each line to obtain RACs.

\section{Pulmonary Function Tests}

FlexiVent, a small animal ventilator, was used to assess respiratory mechanics in anesthetized, mechanically ventilated animals. Mice were ventilated with a tidal volume of $10 \mathrm{ml} / \mathrm{kg}$ and a frequency of $2.5 \mathrm{~Hz}$ following isoflurane anesthesia and i.p. injection of the muscle relaxant pancuronium bromide. The lung function analyses were carried out in two steps: first, pressure-volume $(\mathrm{P}-\mathrm{V})$ loops were obtained by inflating the lungs at increasing pressures up to $33 \mathrm{~cm}$ of $\mathrm{H}_{2} \mathrm{O}$ followed by stepwise deflation at corresponding intervals. Changes in lung resistance and dynamic compliance were measured in response to increasing doses of methacholine (MeCh, Sigma; 0, 3, 6, 12, 25 and $50 \mathrm{mg} / \mathrm{ml}$ in isotonic saline). Resistance and compliance data were collected using a single compartment model and the data plotted after normalizing to percent baseline resistance/compliance ( $\%$ difference $=100 \times($ value-baseline $) /$ baseline $)$ with resistances obtained at $0 \mathrm{mg} / \mathrm{ml}$ of $\mathrm{MeCh}$ for the baseline resistance.

\section{Statistics}

Data are expressed as mean \pm s.e.m. of two to three independent experiments. SigmaStat (Systat Software Inc., San Jose, CA) was used to perform all tests of significance. Student's $t$-test was used for comparisons between two groups and one-way ANOVA with Bonferroni post hoc test for comparisons between more than two groups. Differences were considered statistically significant if $P$-values were $\leqslant 0.05$.

\section{RESULTS \\ Pulmonary Function in Juvenile or Adult Mice Challenged With C. mur at 1 or 7 Days After Birth}

To determine the effect of chlamydial infection in inducing dysfunctional respiratory sequelae, mice were challenged i.n. with C. mur either 1 day (D1) or 7 days (D7) after birth and then studied at the age of 5 or 8 weeks. First, airway resistance in response to escalating doses of $\mathrm{MeCh}$, a cholinergic bronchoconstrictor, was measured. As shown in Figure 1a (left panel), mice infected with C. mur on D1 after birth displayed increased lung resistance in response to escalating doses of inhaled $\mathrm{MeCh}$ compared with age-matched mock (PBS)-treated mice at both 5 weeks (C. mur; $559 \pm 44$, PBS; $407 \pm 82$, at $25 \mathrm{mg} / \mathrm{ml} \mathrm{MeCh}$ ) and 8 weeks (C. mur; $782 \pm 96$, PBS; $370 \pm 118$, at $25 \mathrm{mg} / \mathrm{ml} \mathrm{MeCh}$ ) after challenge. Similarly, D7 infected mice also displayed increased resistance in response to $\mathrm{MeCh}$ challenge at 5 weeks (C. mur; $238 \pm 47$, PBS; $160 \pm 14$, at $25 \mathrm{mg} / \mathrm{ml} \mathrm{MeCh}$ ) with greater resistance by 8 weeks (C. mur; $789 \pm 96$, PBS; $602 \pm 74$ ) after birth (Figure 1c, left panel). No significant difference was observed in baseline airway resistance at 5 or 8 weeks of age between C. mur (mean \pm s.e.m.; $0.69 \pm 0.04 \mathrm{H}_{2} \mathrm{O}$ s/ml) or mock (PBS) infected mice $(0.72 \pm 0.09)$. As expected, the dynamic compliance in response to $25 \mathrm{mg} / \mathrm{ml} \mathrm{MeCh}$ was affected inversely to the dynamic resistance, and was significantly reduced at 8 weeks of age in D1 and D7 challenged mice (Figure 1b and d, right panel). These results suggest that chlamydial infection either during the saccular or alveolar stage of lung development, in the absence of additional triggers of AAD, is sufficient to induce development of AHR as juveniles or adults.

\section{Quasi-Static P-V Curves in Juveniles or Adult Mice Challenged With $C$. mur}

As pulmonary chlamydial infection leads to both peribronchiolar and interstitial inflammation, ${ }^{14}$ quasi-static $\mathrm{P}-\mathrm{V}$ loops were assessed to evaluate incremental lung volumes at 

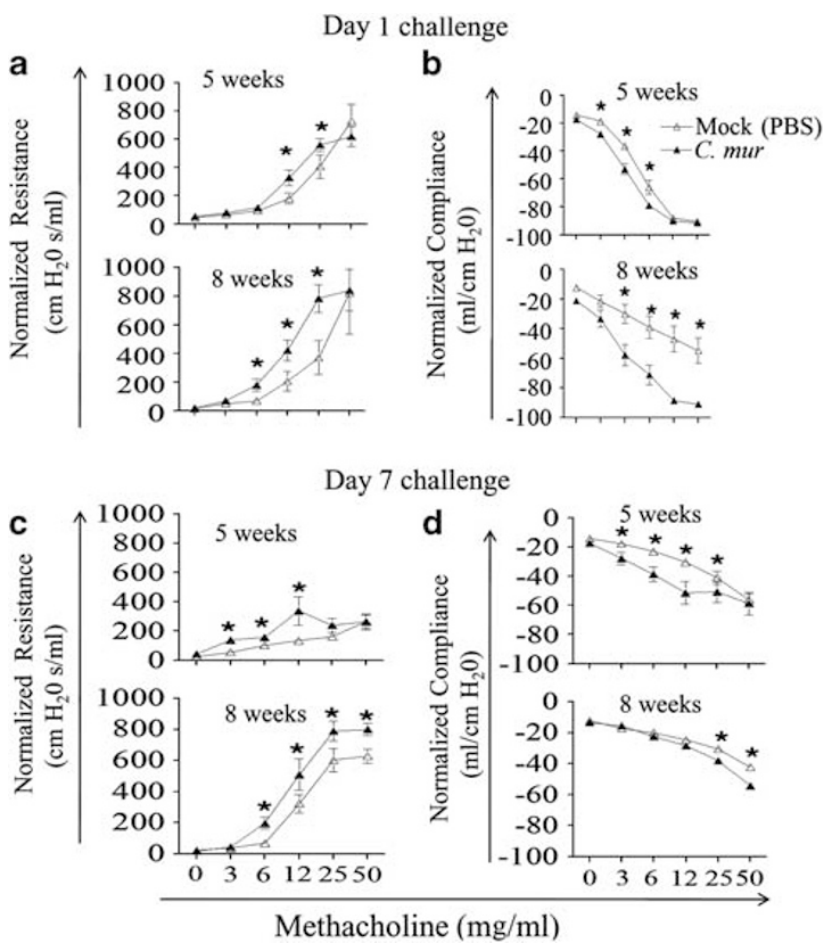

Figure 1 Development of airway dysfunction as juveniles or adults following neonatal pulmonary C. mur infection. Groups $(n=6)$ of pups were challenged with $C$. mur at 1 or 7 days and pulmonary dysfunction was assayed at 5 and 8 weeks after birth. (a) Dynamic resistance (AHR). (b) Dynamic compliance in mice at 5 weeks and 8 weeks with escalating doses of MeCh following C. mur challenge on day 1 after birth. (c, d) AHR and compliance in mice at 5 and 8 weeks following challenge on day 7 after birth. Data are normalized to baseline $(0 \mathrm{mg} / \mathrm{ml}$ of $\mathrm{MeCh})$ and are expressed as mean \pm s.e. and are representative of two independent experiments. ${ }^{*}$ Represents significant differences between $C$. mur infected and mock (PBS) treated groups.

5 or 8 weeks of age in mice challenged on D1 or D7 (Figure 2). As seen in Figure 2, mice challenged with C. mur on D1 or D7 after birth displayed greater lung volumes at both 5 and 8 weeks of age than the corresponding mock (PBS)-challenged mice across the examined pressure range, with significant differences in maximal lung volumes at the greatest examined pressure $\left(33 \mathrm{~cm}\right.$ of $\left.\mathrm{H}_{2} \mathrm{O}\right)$. Specifically, at a pressure of $33 \mathrm{~cm}$ of $\mathrm{H}_{2} \mathrm{O}$, mice challenged with Chlamydia on D1 displayed significantly greater mean lung volumes $(0.45 \pm 0.02 \mathrm{ml} / \mathrm{g}$ at 5 weeks and $0.79 \pm 0.01 \mathrm{ml} / \mathrm{g}$ at 8 weeks $)$ than age-matched mock-infected mice $(0.36 \pm 0.01 \mathrm{ml} / \mathrm{g}$ at 5 weeks and $0.68 \pm 0.01 \mathrm{ml} / \mathrm{g}$ at 8 weeks; Figure $2 \mathrm{a}$ and c). Similarly, maximal lung volumes were significantly increased at 5 and 8 weeks in D7 challenged mice $(0.59 \pm 0.01 \mathrm{ml} / \mathrm{g}$ at 5 weeks and $0.78 \pm 0.01 \mathrm{ml} / \mathrm{g}$ at 8 weeks) compared with age-matched mock-infected mice $(0.54 \pm 0.01 \mathrm{ml} / \mathrm{g}$ at 5 weeks and $0.73 \pm 0.01 \mathrm{ml} / \mathrm{g}$ at 8 weeks) as seen in Figure $2 \mathrm{~b}$ and $\mathrm{d}$. It appeared that chlamydial challenge on D1 induced more pronounced effects on normalized lung volumes compared with D7. Collectively, these results suggest that chlamydial challenge on either D1 or D7 leads to greater maximal lung volume, suggesting increased maximal tissue distensibility as juveniles or adults, despite decreased dynamic compliance at normal tidal volumes when compared with mock-challenged mice.

\section{Pulmonary Histopathology in Juvenile or Adult Mice Challenged With C. mur}

To further explore the structural basis for the increased distensibility, but decreased dynamic compliance as sequelae to $C$. mur infection as neonates, qualitative examination of H\&E-stained tissue sections by microscopy and determinations of quantitative RACs at 5 and 8 weeks of age following $\mathrm{D} 1$ or D7 challenge were performed. As shown in Figure $3 \mathrm{~b}$ and e, mice challenged with Chlamydia on D1 displayed larger alveoli compared with mock (PBS)-treated mice at both 5 and 8 weeks (Figure 3a and d). Similar observations made in mice infected with C. mur on D7 after birth also revealed larger alveoli compared with mock (PBS)-treated mice at 5 weeks (Figure 3c) but not at 8 weeks (Figure 3f). We further extended these observations to quantify the number of alveoli by performing RACs (Figure 4). These analyses revealed that mice infected with C. mur on D1 displayed fewer and larger alveoli at both 5 weeks (Figure 4a) and 8 weeks (Figure 4c) compared with mock (PBS)-treated mice. In addition, D7 challenged animals displayed fewer alveoli at 5 weeks (Figure 4b), but not at 8 weeks (Figure 4d), when compared with age-matched mock-challenged animals. These results suggest that neonatal chlamydial infection, particularly at D1, leads to alterations in alveolar structure and loss of lung parenchyma, thus enhancing over distension of alveoli in juvenile and adult life.

\section{Effect of Antibiotic Treatment Following C. mur Challenge on Day 1 After Birth on Chlamydia-Induced Altered Lung Function as Adults}

Given that neonatal chlamydial lung infection leads to chronic respiratory sequelae, and that erythromycin (Ery) is widely used to treat the pneumonia during acute infection, we evaluated whether this antimicrobial had a beneficial effect on the long-term sequelae in neonatally challenged mice. Neonatal mice were challenged i.n. with $C$. mur or PBS (mock) on D1 or D7 followed 48 h later by i.p. Ery treatment for 3 days. As shown in Figure $5 \mathrm{a}$ and $\mathrm{b}$, the pulmonary chlamydial clearance induced by Ery treatment was confirmed. Specifically, C. mur challenged pups treated with Ery (C. $m u r+$ Ery) exhibited a significantly reduced number of chlamydial IFUs in the lungs within 2 days of antibiotic administration (Figure 5a) and complete clearance of C. mur after 3 days of antibiotic administration (Figure 5b). Following complete bacterial clearance, C. mur + Ery mice were rested and lung function analyses were carried out at 8 weeks of age. As shown in Figure 6, D1 C. mur-challenged mice displayed greater resistance in response to $25 \mathrm{mg} / \mathrm{ml}$ MeCh compared with age-matched mock (PBS)-treated mice (Figure 6a). C. mur-infected and Ery-treated (C. mur + Ery) 


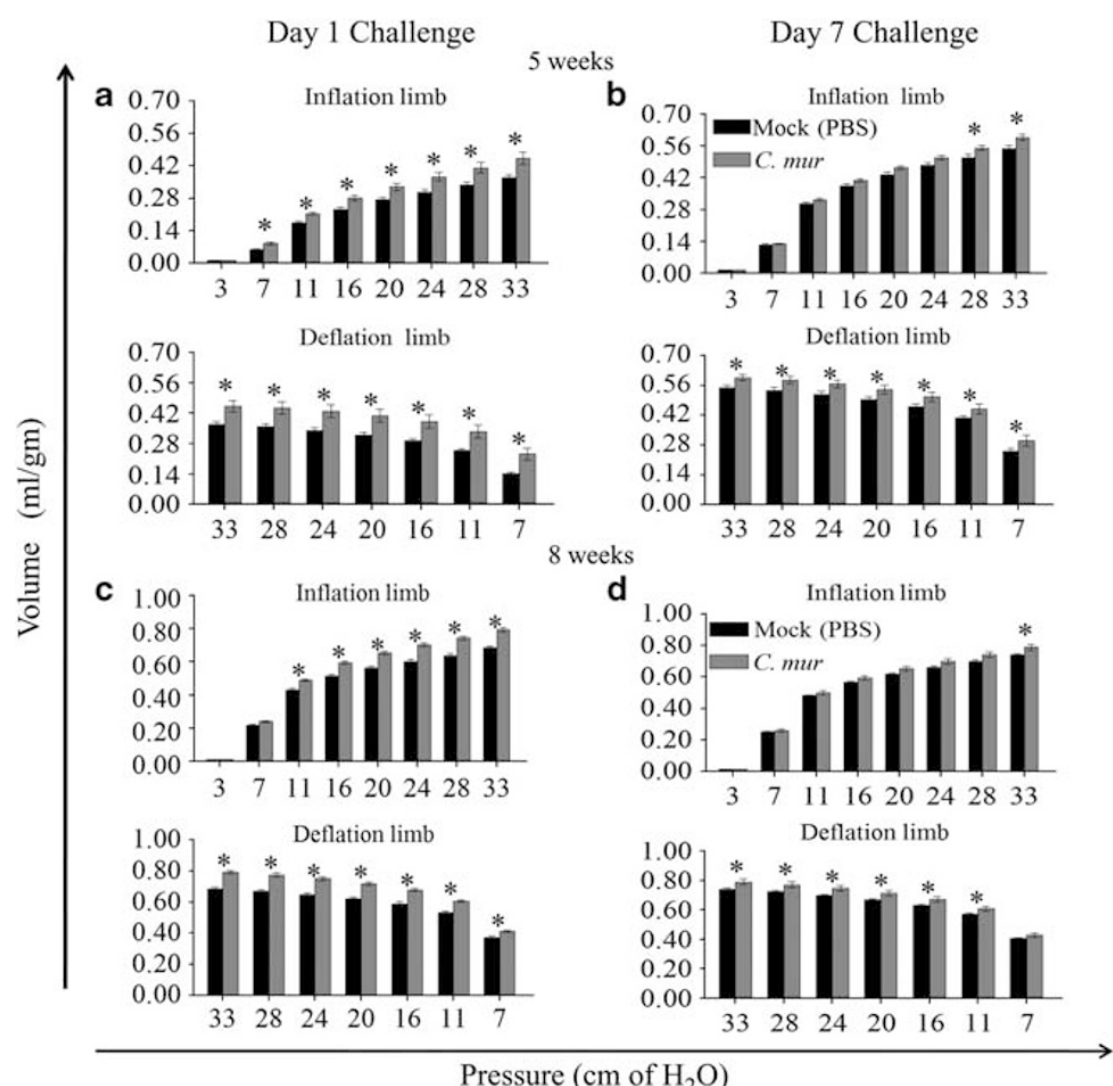

Figure 2 Alterations of lung parenchymal function as juveniles or adults following neonatal pulmonary C. mur infection. Groups ( $n=6$ ) of pups were infected with C. mur on day 1 or 7 and quasi-static pressure-volume loops were measured at 5 or 8 weeks after birth. P-V loops determined by the forced oscillation technique are plotted as mean \pm s.e. at each inflation/deflation pressure. $(\mathbf{a}, \mathbf{c})$ Volume $(\mathrm{ml})$ in the lung at each increasing/decreasing pressure (inflation/deflation limb) at 5 or 8 weeks after $C$. mur challenge on day 1 after birth. (b, d) Volume (ml) in the lung at each increasing/decreasing pressure (inflation/deflation limb) at 5 or 8 weeks after challenge on day 7 after birth. The results are representative of two independent experiments. ${ }^{*}$ Represents significant differences between volume of air in $\mathrm{ml}$ between $C$. mur infected mice and mock (PBS) treated mice.

mice exhibited a significant reduction in airway resistance, but still had significantly more AHR in response to $25 \mathrm{mg} / \mathrm{ml}$ MeCh challenge than mock (PBS) controls (Figure 6a). As a control, mock-challenged mice treated with Ery alone (Ery) exhibited similar resistance to that of mock (PBS)treated mice (data not shown). Similar trends of greater AHR also were observed at 8 weeks of age in D7 C. mur-challenged mice, with C. mur infected and Ery treatment (C. mur + Ery) significantly reducing the airway resistance on $\mathrm{MeCh}$ challenge to levels comparable with that in age-matched mock-challenged animals (Figure $6 \mathrm{~b}$ ). These results suggest that Ery administration following neonatal challenge with Chlamydia during the saccular and alveolar stage of lung development leads to reduction in AHR as adults, although not to the levels of control mice.

$\mathrm{P}-\mathrm{V}$ curves were measured on the FlexiVent using the forced oscillation technique. As shown in Figure 7, D1 C. mur-challenged mice once again displayed greater incremental lung volumes across the examined pressure range compared with age-matched mock (PBS)-challenged mice (Figure $7 \mathrm{a}$ and $\mathrm{c}$ ). Erythromycin treatment following C. mur challenge significantly reduced lung volumes compared with lungs of $C$. mur-challenged mice that did not receive antibiotics, but not to the volumes recorded in the mock (PBS) controls (Figure $7 \mathrm{a}$ and $\mathrm{c}$, left panel). Unchallenged mice treated with Ery alone exhibited similar lung volumes to those measured in mock (PBS)-challenged mice (data not shown). In D7 C. mur-challenged mice at 8 weeks of age, Ery treatment only led to significant reductions in maximal lung volumes down to levels seen in age-matched mockchallenged animals (Figure $7 \mathrm{a}$ and d, right panel). These results suggest that Ery administration following neonatal C. mur challenge leads to reduced lung volumes as adults, although not to the levels in control mice, when the mice were challenged during the saccular stage of lung development.

RAC analyses also were performed on Ery-treated mice at 8 weeks following day 1 or 7 chlamydial challenge. As shown in Figure $8 \mathrm{a}$, mice challenged with $C$. mur on D1 displayed reduced numbers of alveoli at 8 weeks of age compared with age-matched mock (PBS)-treated mice as previously observed (Figure 4). In comparison, Ery-treated C. murchallenged mice had significantly greater numbers of alveoli 

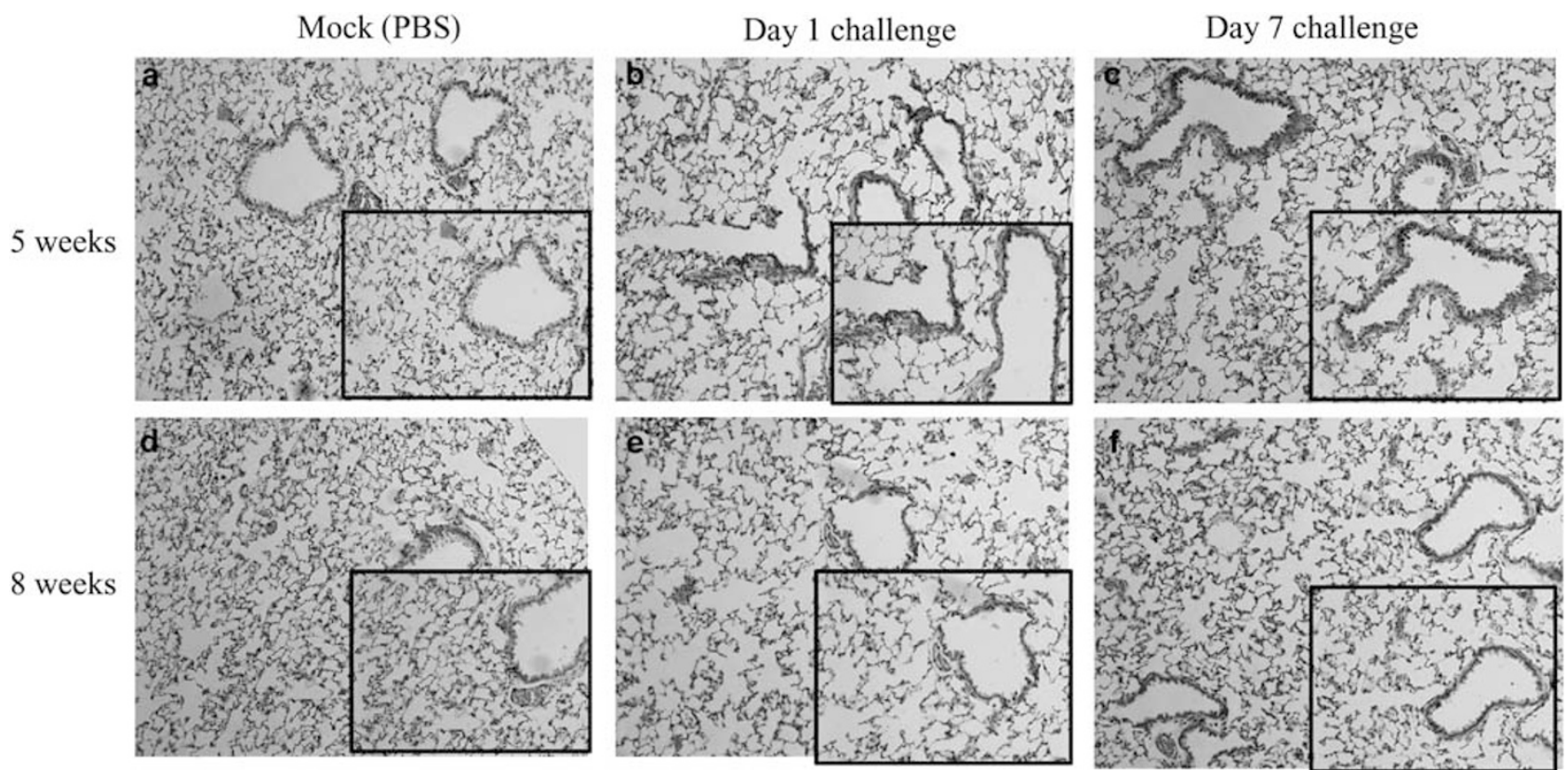

Figure 3 Pulmonary histopathology at 5 or 8 weeks following neonatal C. mur infection. Groups $(n=6)$ of pups were infected with C. mur on day 1 or 7 and qualitative histological evaluation was performed at 5 or 8 weeks after birth demonstrates qualitative images on lung sections at $\times 100$ magnification. The slides $\mathbf{b}$ and $\mathbf{e}$ demonstrate representative images at 5 and 8 weeks after day 1 challenge, respectively, and $\mathbf{c}$ and $\mathbf{f}$ demonstrate lung sections at 5 and 8 weeks following day 7 challenge. Representative lung sections from age-matched mock (PBS)-treated mice are shown in a and $\mathbf{d}$. The inserts show high resolution images at the corresponding time points. Results are representative of two independent experiments.

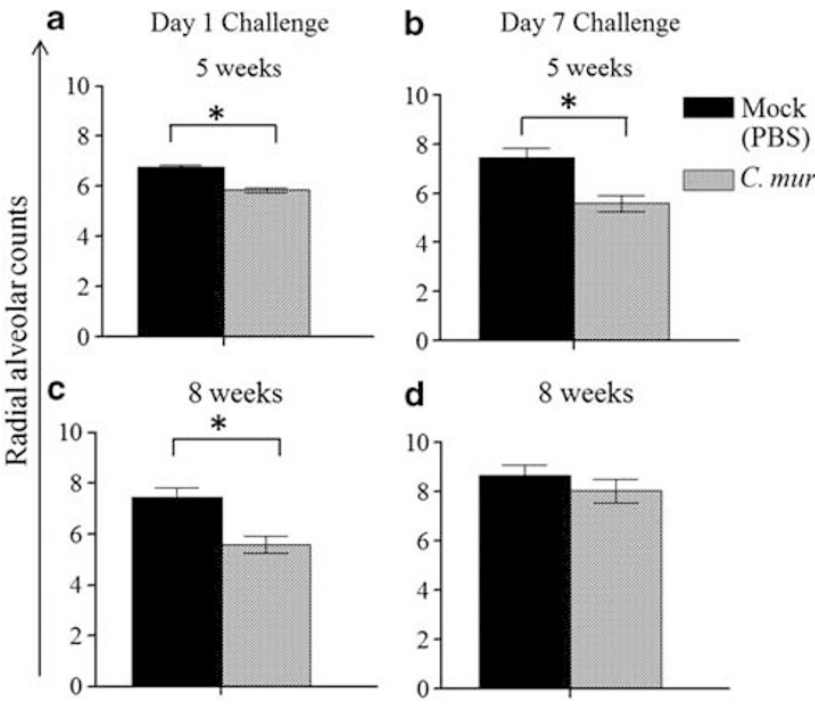

Figure 4 Radial alveolar counts at 5 or 8 weeks following neonatal pulmonary C. mur challenge. Groups $(n=6-7)$ of 1 or 7 day old pups were challenged with $C$. mur and rested for 5 or 8 weeks followed by determination of the RACs. (a, c) The RAC at 5 and 8 weeks following day 1 C. mur challenge. (b, d) The RAC at 5 and 8 weeks following challenge on day 7 after birth. Results are expressed as mean \pm s.e. and are representative of two independent experiments. ${ }^{*}$ Represents significant differences in RAC between mock (PBS) treated and C. mur infected mice.

compared with untreated C. mur-challenged mice. Similar analysis performed on D7 challenged mice (Figure 8b) revealed that Ery treatment had a minimal effect on the

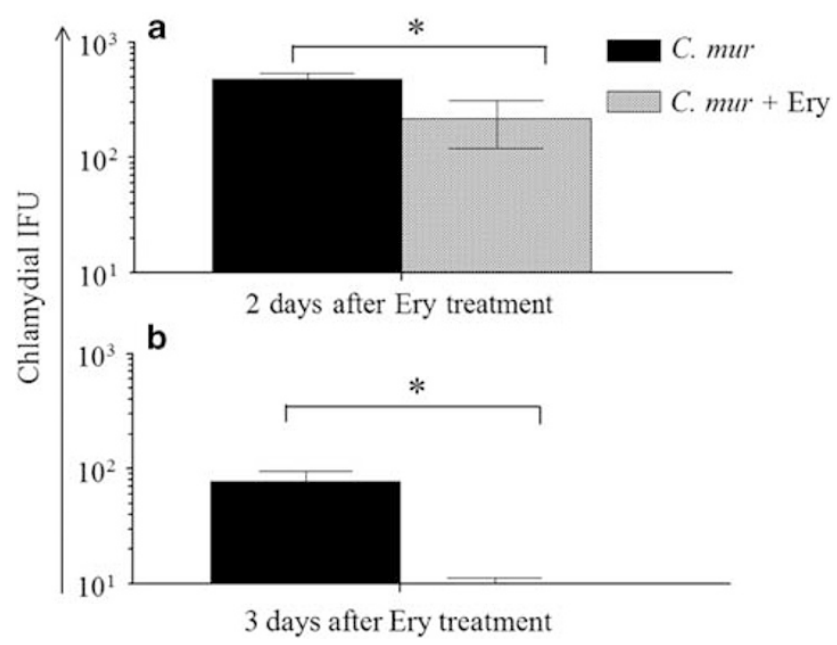

Figure 5 Effect of antimicrobials on bacterial clearance in the lung following neonatal pulmonary chlamydial challenge. Groups $(n=4)$ of pups were challenged i.n with $C$. mur on day 1 after birth and the reduction in bacterial burden was confirmed by plating the lung homogenates on HeLa cell monolayers after 2 (a) and 3 (b) days of antimicrobial administration. Results are expressed as mean \pm s.e. are representative of two independent experiments. ${ }^{\star}$ Represents significant differences in bacterial burden between C. mur infected vs C. mur infected + Ery treated mice.

numbers of alveoli in comparison with untreated mice challenged with $C$. mur These results suggest that early intervention with Ery leads to restoration of lung structure 
when mice were challenged during the saccular stage of lung development.

\section{DISCUSSION}

C. trachomatis infections are known to induce post-infection sequelae, including trachoma and pelvic inflammatory
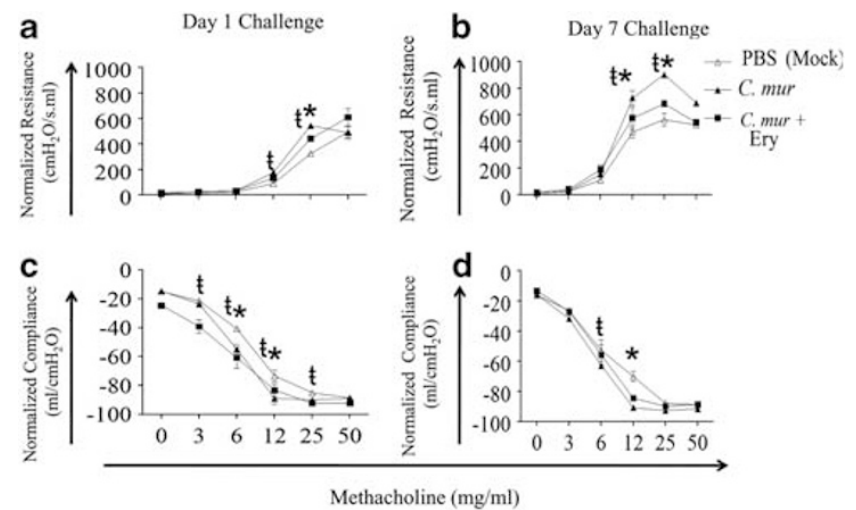

Figure 6 Pulmonary function in mice at 8 weeks following antimicrobial administration as neonates. Groups $(n=6)$ of mice were challenged with C. mur on day 1 or 7 after birth, and changes in AHR were measured at 8 weeks following challenge. (a, c) Dynamic resistance/compliance in mice treated with Ery following challenge on day 1 after birth. (b, d) Dynamic resistance/compliance in mice treated with Ery following i.n. C. mur challenge on day 7 after birth. Asterisks $\left(^{*}\right)$ represents significance between $C$. mur and C. mur + Ery-treated groups and tau $\left({ }^{\tau}\right)$ represents significance between $C$. mur and mock (PBS)-treated mice. Results are expressed as mean \pm s.e. and are representative of two independent experiments. disease, ${ }^{21}$ all arising from the host inflammatory response to infection that induces collateral damage. ${ }^{22}$ Perinatal transmission of C. trachomatis has been known to cause neonatal pneumonia, ${ }^{23-25}$ and also has been correlated with the development of respiratory dysfunction in later life, long after resolution of the neonatal infection. ${ }^{6}$ In this study, we used a mouse model of neonatal C. mur infection to demonstrate a causal association between neonatal pulmonary chlamydial infection and respiratory dysfunction, including airway and parenchymal, in juvenile and adult life. We also found that infection occurring during the saccular stage of lung development may have more severe consequences than those occurring during the alveolar stage of development, suggesting that infection occurring in an immature neonatal lung may lead to more significant later life respiratory consequences than infection that occurs in a more mature lung. Furthermore, Ery, a drug of choice for treatment of neonatal chlamydial pneumonia, was found to be partially effective in reducing the post-infection sequelae.

Post-infection sequelae following neonatal pulmonary chlamydial infection in a mouse model has been documented in several recent studies. ${ }^{16}$ Specifically, it was shown that neonatal chlamydial infection can differentially drive key features of AAD, including the induction of both Th1 and Th2 responses to the unrelated Th2 inducing antigen ovalbumin. $^{16}$ Furthermore, pulmonary chlamydial infection induced exacerbation of AAD in response to subsequent ovalbumin sensitization/challenge was reversed by the blockage of neutrophilic infiltration into the lungs. ${ }^{26}$

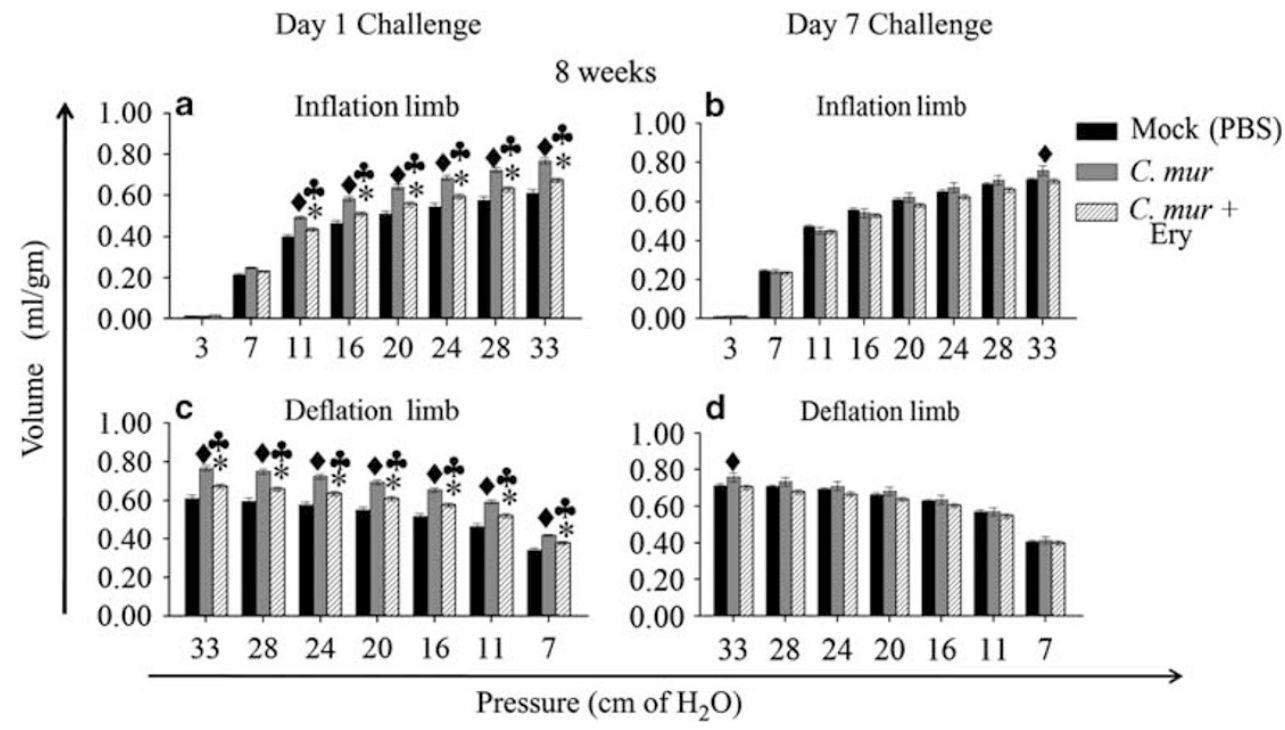

Figure 7 Effect of antimicrobials on altered lung function following C. mur infection. Groups $(n=6)$ of pups challenged i.n. with C. mur on day 1 or 7 after birth, treated with Ery and rested before performing PV loops at 5 or 8 weeks after challenge. (a, c) Volume (ml) recruited in the lung at various increasing/ decreasing pressures (inflation/deflation limb) at 8 weeks following day 1 challenge and Ery treatment. (b, d) Volume (ml) recruited at various pressures (inflation/deflation limb) at 8 weeks following day 7 challenge and Ery treatment. Results are expressed as mean \pm s.e. and are representative of two independent experiments. $\downarrow$ Represents significant differences between mock (PBS) treated vs C. mur infected mice. Represents significant differences between C. mur infected vs C. mur infected + Ery treated groups. * Represents significant differences between mock (PBS) treated group vs $C$. mur + Ery treated groups. 

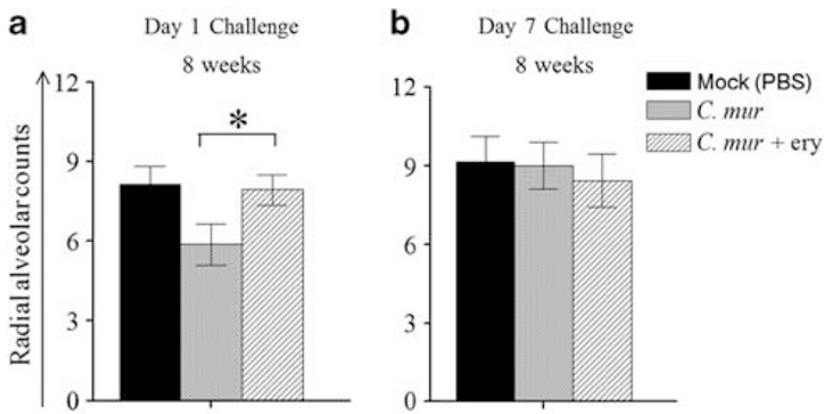

Figure 8 Effect of antimicrobials on lung structure as adults following neonatal $C$. mur infection. Groups $(n=6)$ of pups challenged i.n. with C. mur on day 1 or 7 after birth and treated with Ery were rested before performing RAC at 8 weeks after challenge. (a) RAC counts at 8 weeks following day 1 C. mur challenge and Ery treatment. (b) RACs at 8 weeks following day 7 C. mur challenge and Ery treatment. Results are expressed as mean \pm s.e. ${ }^{*} P=0.05$ is considered significant.

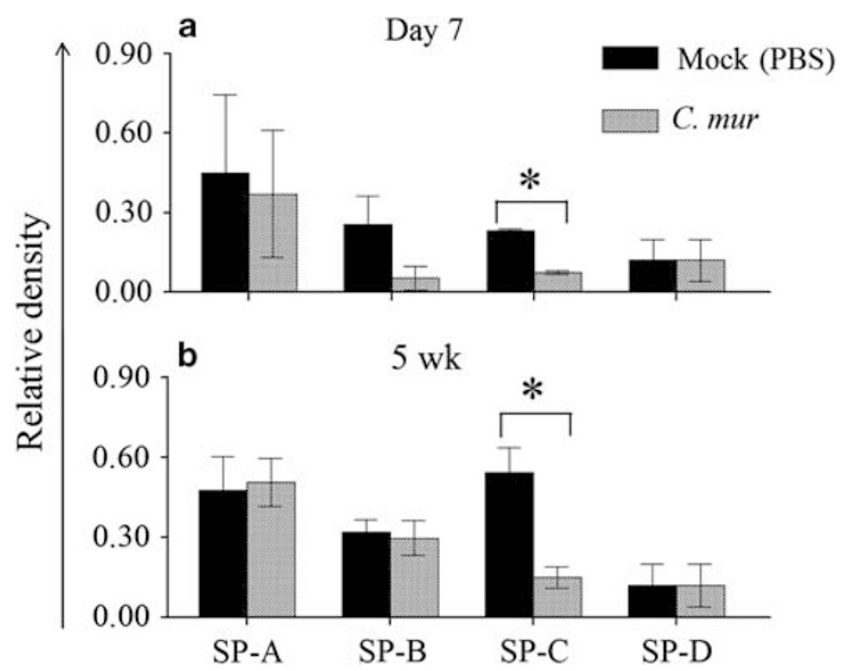

Figure 9 Pulmonary surfactant protein expression following neonatal C. mur infection. Groups $(n=4)$ of pups were challenged i.n. with C. mur on day 1 after birth and lungs were collected on day 7 (a) or 5 weeks (b) after challenge to determine alterations in surfactant expression by western blotting analysis. Results are expressed as mean \pm s.e. and are representative of two independent experiments. ${ }^{*}$ Represents significant differences in SP-C expression between C. mur infected vs mock (PBS) treated mice at the corresponding time intervals.

Although the neutrophilic infiltration was associated with Th1/Th17 responses, the blockade of IFN- $\gamma$ or IL-17 did not significantly alter the ovalbumin-induced AAD, suggesting that factors other than these cytokines may have a crucial role in the process. ${ }^{26}$

The mechanistic basis of chlamydial neonatal pulmonary infection-induced changes to subsequent events in adult life such as allergen exposure may be unraveled by primarily understanding the effects of infection per se on lung structure and function. Although the majority of the characterization in earlier studies focused on ovalbumin-induced AAD, the authors made anecdotal observations that the neonatal infection per se, in the absence of additional ovalbumin sensitization/challenge, led to induction of significantly enhanced AHR and alveolar diameter. ${ }^{17}$ We now confirm and extend these observations by detailed experiments demonstrating that neonatal pulmonary chlamydial infection leads to chronic sequelae in juvenile and adult life long after clearance of the bacterium, as evidenced by enhanced AHR, reduced RACs and increased lung compliance, collectively suggesting emphysematous changes. These effects were evident both at the age of 5 weeks (juvenile) and 8 weeks (adult) in mice challenged neonatally with Chlamydia in the pulmonary compartment, suggesting that the changes induced by the neonatal infection are long lasting, and may be permanent. Interestingly, we also observed significant reduction in expression of surfactant protein $\mathrm{C}$ during the acute phase of the infection (day 7) and at 5 weeks following C. mur challenge on day 1 after birth (Figure 9). In parallel analyses, differences in expression of various enzymes such as esterases, alkaline phosphatases and lipases (Supplementary Table 1) were observed at 5 weeks following chlamydial clearance in mice infected on day 1 after birth compared with mockchallenged animals, using API-ZYM assays (Supplementary Information). Thus, in addition to the chronic modulation of inflammatory cytokines such as IFN- $\gamma$ and IL-17 as shown in the C. mur-ovalbumin AAD model, ${ }^{26}$ the neonatal pulmonary chlamydial infection also may affect the levels of surfactant proteins that are involved in maintenance of lung structure and function, ${ }^{27-31}$ and various biochemical enzymes that could differentially induce inflammatory responses in the lung (Figure 9). ${ }^{31,32}$

Chlamydial infection in mice at 1 day after birth induced more severe respiratory sequelae at 8 weeks of age compared with infection at 7 days after birth. The neonatal mouse lung at 1 day after birth is in the saccular stage of development, whereas the 7-day-old mouse lung is in the alveolar stage of development. ${ }^{33}$ This suggests that respiratory sequelae following chlamydial infection in an immature lung may be more severe than the infection that occurs in a matured lung. Our results are in accordance with Horvat et $a l,{ }^{17}$ who showed that early life (neonatal; day 1) respiratory C. mur infections result in greater altered lung structure, function and enhanced AHR compared with infection that occurs in later life. These results suggest that the environment of the neonatal lung is a major determinant of disease patterns in later life. In this context, Culley et $a l^{34}$ showed that the age at which primary viral infection occurs determines the pattern of T-cell-mediated disease during re-infection in adulthood in a mouse model of RSV infection.

The macrolide antimicrobial Ery is an approved treatment for symptomatic neonatal C. trachomatis pneumonia (CDC, 2010 STD Treatment Guidelines; cdc.gov/std/treatment/ 2010/chlamydial-infections.html). Macrolides have been shown to reduce neutrophil chemotaxis, oxidative burst and proinflammatory cytokine generation in various models. ${ }^{35}$ 
Macrolides also reduce mucus hypersecretion in patients with diffuse panbronchiolitis, chronic bronchitis and sinus disease. ${ }^{35}$ Evidence suggests that macrolides may protect the epithelium from attack by bioactive phospholipids generated as part of the inflammatory process. ${ }^{36,37}$ In our study, Ery treatment of Chlamydia-infected neonatal mice promoted the clearance of infection from the lungs within 3 days. This early elimination of the infection also was partially effective in reducing the respiratory sequelae in adult life as shown by RACs, which demonstrated that Ery administration restored Chlamydia induced reduced alveoli seen in adults. These findings suggest that the usage of antibiotics to treat neonatal chlamydial pneumonia may have important benefits beyond the alleviation of the acute pneumonia. However, the complete set of adverse effects of neonatal chlamydial infection may not be alleviated by administration of antibiotics, although it cannot be excluded that usage of a different antibiotic and/or regimen may be more efficacious in preventing the chronic sequelae. In addition, asymptomatic chlamydial pulmonary infections have been described ${ }^{25}$ and as a consequence, infected neonates may not receive treatment and grow into adulthood with respiratory dysfunction. As with other animal models of human disease, results from our model of $C$. mur pulmonary infection of neonatal mice and subsequent respiratory dysfunction in later life may not be directly extrapolated to humans. For example, C. trachomatis has been shown to use different mechanisms to evade the host IFN- $\gamma$ response in humans, when compared with C. $m u r$ in mice, ${ }^{38}$ and these organisms have certain differences, such as the shorter life cycle of $C$. mur (approximately $24 \mathrm{~h} v s 48-72 \mathrm{~h}$ for $C$. trachomatis) as well as differences in pathogenesis due to differences in development and life span of their respective hosts, despite several striking similarities between the murine and human organisms. ${ }^{39}$ Although our results from the C. mur infection in neonatal mice may not be directly extrapolated to those of $C$. trachomatis infections in humans (owing to the different life cycles and pathogenesis), it provides a useful animal model to study the serious nature of the potential sequelae of early chlamydial respiratory infection and lends justification to further detailed mechanistic investigations. It also highlights the importance of prenatal screening and treatment of pregnant women for $C$. trachomatis in order to prevent the possible long-lasting effects of neoanatal pulmonary chlamydial infections.

Supplementary Information accompanies the paper on the Laboratory Investigation website (http://www.laboratoryinvestigation.org)

\section{ACKNOWLEDGEMENT}

This work is supported by the National Institutes of Health Grant 1 RO1 Al074860.

\section{DISCLOSURE/CONFLICT OF INTEREST}

The authors declare no conflict of interest.
1. Chawla R, Bhalla P, Sachdev H. A pilot study of Chlamydia trachomatis pneumonia in infants. Indian J Med Microbiol 2004;22:185-187.

2. Were F, Govedi A, Revathi G, et al. Chlamydia as a cause of late neonatal pneumonia at Kenyatta National Hospital, Nairobi. East Afr Med J 2002;79:476-479.

3. Chen C, Wu K, Tang R, et al. Characteristics of Chlamydia trachomatis infection in hospitalized infants with lower respiratory tract infection. J Microbiol Immunol Infect 2007:40:255-259.

4. Beem M, Saxon E. Respiratory-tract colonization and a distinctive pneumonia syndrome in infants infected with Chlamydia trachomatis. N Engl J Med 1977;296:306-310.

5. Beem M, Saxon E, Tipple M. Treatment of chlamydial pneumonia of infancy. Pediatrics 1979;63:198-203.

6. Weiss S, Newcomb R, Beem M. Pulmonary assessment of children after chlamydial pneumonia of infancy. J Pediatr 1986;108(5 part 1): 659-664.

7. Cunningham $C$, Johnston $S$, Julious $S$, et al. Chronic Chlamydia pneumoniae infection and asthma exacerbations in children. Eur Respir J 1998;11:345-349.

8. Esposito S, Blasi F, Arosio C, et al. Importance of acute Mycoplasma pneumoniae and Chlamydia pneumoniae infections in children with wheezing. Eur Respir J 2000;16:1142-1146.

9. Blasi F. Atypical pathogens and respiratory tract infections. Eur Respir J 2004;24:171-181.

10. Kumar S, Hammerschlag M. Acute respiratory infection due to Chlamydia pneumoniae: current status of diagnostic methods. Clin Infect Dis 2007:44:567-576.

11. Darville T. Chlamydia trachomatis infections in neonates and young children. Semin Pediatr Infect Dis 2005;16:235-244.

12. Roseman $M$, Mahon B, Downs $S$, et al. Oral erythromycin prophylaxis vs watchful waiting in caring for newborns exposed to Chlamydia trachomatis. Arch Pediatr Adolesc Med 2003;157:565-571.

13. Webley W, Tilahun Y, Lay K, et al. Occurance of Chlamydia trachomatis and Chlamydia pneumoniaein pediatric respiratory infections. Respir J 2009:33:360-367.

14. Jupelli $M$, Guentzel $M$, Meier $P$, et al. Endogenous IFN-gamma production is induced and required for protective immunity against pulmonary chlamydial infection in neonatal mice. J Immunol 2008;180:4148-4155.

15. Jupelli M, Selby D, Guentzel M, et al. The contribution of interleukin12 /interferon-gamma axis in protection against neonatal pulmonary Chlamydia muridarum challenge. J Interferon Cytokine Res 2010; 30:407-415.

16. Horvat J, Beagley $\mathrm{K}$, Wade $\mathrm{M}$, et al. Neonatal chlamydial infection induces mixed T-cell responses that drive allergic airway disease. Am J Respir Crit Care Med 2007;176:556-564.

17. Horvat J, Starkey M, Kim R, et al. Early-life chlamydial lung infection enhances allergic airways disease through age-dependent differences in immunopathology. J Allergy Clin Immunol 2010;125:617-625, 625.e611-625.e616.

18. Abdelrahman $Y$, Belland $R$. The chlamydial developmental cycle. FEMS Microbiol Rev 2005;29:949-959.

19. Cooney T, Thurlbeck W. The radial alveolar count method of Emery and Mithal: a reappraisal 1-postnatal lung growth. Thorax 1982;37: 572-579.

20. Cooney T, Thurlbeck W. The radial alveolar count method of Emery and Mithal: a reappraisal 2-intrauterine and early postnatal lung growth. Thorax 1982;37:580-583.

21. Darville $T$, Hiltke $T$. Pathogenesis of genital tract disease due to Chlamydia trachomatis. J Infect Dis 2010;201(Suppl 2):S114-S125.

22. Brunham R, Rey-Ladino J. Immunology of Chlamydia infection: implications for a Chlamydia trachomatis vaccine. Nat Rev Immunol 2005; 5:149-161.

23. Gencay $M$, Koskiniemi $M$, Fellman V, et al. Chlamydia trachomatis infection in mothers with preterm delivery and in their newborn infants. APMIS 2001;109:636-640.

24. Darville T. Chlamydia trachomatis genital infection in adolescents and young adults. Adv Exp Med Biol 2006;582:85-100.

25. Jain S. Perinatally acquired Chlamydia trachomatis associated morbidity in young infants. J Matern Fetal Med 1999;8:130-133.

26. Horvat J, Starkey M, Kim R, et al. Chlamydial respiratory infection during allergen sensitization drives neutrophilic allergic airways disease. J Immunol 2010;184:4159-4169. 
27. Bland R. Neonatal chronic lung disease in the post-surfactant era. Biol Neonate 2005;88:181-191.

28. Ferrer-Roca O, Gotzens V, Ruano-Gil D, et al. Alveolar development of the lung of the rat. Part I. Arch Ital Anat Embriol 1985;90: 91-99.

29. Ferrer-Roca O, Gotzens V, Ruano-Gil D, et al. Alveolar development of the lung of the rat. Part II. Morphogenesis of surfactant. Arch Ital Anat Embriol 1985;90:101-110.

30. Hohlfeld J, Fabel H, Hamm $\mathrm{H}$. The role of pulmonary surfactant in obstructive airways disease. Eur Respir J 1997;10:482-491.

31. Harrison $H$, English $M$, Lee $C$, et al. Chlamydia trachomatis infant pneumonitis: comparison with matched controls and other infant pneumonitis. N Engl J Med 1978;298:702-708.

32. Lian $X$, Yan C, Yang L, et al. Lysosomal acid lipase deficiency causes respiratory inflammation and destruction in the lung. Am J Physiol Lung Cell Mol Physiol 2004;286:L801-L807.

33. Perl A, Whitsett J. Molecular mechanisms controlling lung morphogenesis. Clin Genet 1999;56:14-27.
34. Culley F, Pollott J, Openshaw P. Age at first viral infection determines the pattern of $T$ cell-mediated disease during reinfection in adulthood. J Exp Med 2002;196:1381-1386.

35. Sharma S, Jaffe A, Dixon G. Immunomodulatory effects of macrolide antibiotics in respiratory disease: therapeutic implications for asthma and cystic fibrosis. Paediatr Drugs 2007;9:107-118.

36. Tamaoki J, Kadota J, Takizawa H. Clinical implications of the immunomodulatory effects of macrolides. Am J Med 2004;117(Suppl 9A): $5 \mathrm{~S}-11 \mathrm{~S}$.

37. Takizawa $H$, Desaki $M$, Ohtoshi $T$, et al. Erythromycin suppresses interleukin 6 expression by human bronchial epithelial cells: a potential mechanism of its anti-inflammatory action. Biochem Biophys Res Commun 1995;210:781-786.

38. Nelson D, Virok $D$, Wood $H$, et al. Chlamydial IFN-gamma immune evasion is linked to host infection tropism. Proc Natl Acad Sci USA 2005;102:10658-10663.

39. McClarty G, Caldwell H, Nelson D. Chlamydial interferon gamma immune evasion influences infection tropism. Curr Opin Microbiol 2007;10:47-51. 